\title{
Joint Prevention and Control of New Coronavirus Infection in Chengdu Chongqing Urban Agglomeration
}

\author{
Menghan Huang \\ School of Public Administration and Political Science and Law, Southwest Jiaotong University, Chengdu, \\ China \\ 734164050@qq.com
}

Keywords: Urban management, Urban agglomeration, Traffic flow, Population migration flow, Regional prevention and control

\begin{abstract}
Under the background of the network of urban agglomeration, the population migration flow and traffic flow network with the central metropolis as the core gradually form in the urban agglomeration. The occurrence of major public health events (such as the spread of new coronavirus) is closely related to the distribution of traffic flow and population migration flow in urban agglomerations. The spread and transmission of the virus and the risk level of urban epidemic are affected by the former. Therefore, this paper analyzes the epidemic characteristics of new coronavirus in Chengdu Chongqing urban agglomeration from the perspective of traffic flow and population migration flow, and puts forward some joint prevention and control suggestions from the perspective of urban agglomeration, so as to improve the ability and level of urban agglomeration to cope with major public health emergencies.
\end{abstract}

\section{Introduction}

The novel coronavirus pneumonia novel coronavirus pneumonia has become a "public health emergencies of international concern" since the first confirmed case of new crown pneumonia in late 2019. The epidemic spread from Wuhan to the whole province and even the whole country through convenient transportation network. Based on the analysis of population migration flow across provinces, this paper finds that there is a close relationship between Chengdu Chongqing urban agglomeration and Wuhan. The spread and spread of epidemic is closely related to traffic flow and population migration. Based on the analysis of traffic flow and population migration flow in Chengdu Chongqing urban agglomeration, this paper studies the regional distribution and transmission characteristics of epidemic situation in Chengdu Chongqing urban agglomeration, and outlines and analyzes the relevant practices and experience of joint deployment of epidemic prevention and control in Chengdu Chongqing urban agglomeration.

\section{Risk Analysis of Chengdu Chongqing Urban Agglomeration}

As the main form of urbanization, urban agglomeration is the most dynamic spatial organization in the current national economic activities. The comprehensive transportation and the accessibility of information network in urban agglomerations make the cities in urban agglomerations form a relatively complete urban agglomeration. How to effectively construct cross regional joint prevention and control in the face of major public health events has become the focus of improving the quality of modern urban governance. Based on the importance of urban agglomerations in the prevention of this major health event, when studying how to establish a good prevention and control mechanism, the research perspective is expanded from "point" to "face", mainly focusing on the urban agglomeration of Chengdu, Deyang, Mianyang and other 15 prefecture level cities The regional distribution characteristics of the epidemic situation in 29 districts and counties of Chongqing were discussed. 


\subsection{Characteristics of Traffic Flow in Urban Agglomeration}

Due to the rapid development of transportation and information technology, the traditional concept of "local space" and "place space" are not enough to describe the existing social space form. Therefore, the famous sociologist Manuel Castells proposed the "flow space" theory ${ }^{[1-2]}$. "Flow space" can reflect the flow of elements between cities, and truly describe the relationship between cities' mutual exchange and cooperation. In the study of urban relations, the data of inter city relationship flow can be quantified, which can be divided into tangible flow and intangible flow. Tangible flow includes: people flow, logistics flow and traffic flow; intangible flow includes: industrial flow, capital flow, information flow, etc. ${ }^{[3]}$. As an important component of the "tangible flow", traffic flow is the main carrier of various elements flow transmission, which can reflect the commuting and business communication between urban nodes in the region, and is the comprehensive embodiment of communication links between cities ${ }^{[3]}$.

Chengdu Chongqing urban agglomeration has formed its own unique "flow space" form. Chengdu occupies an absolute dominant position in the transportation network of Chengdu Chongqing urban agglomeration, forming a radial network with surrounding Deyang, Mianyang, Suining, Ziyang, Ya'an, Meishan and Leshan, which indicates that the planning and implementation of Chengdu Plain urban agglomeration has strengthened the traffic links between Chengdu and surrounding cities, and four major axis belts have been formed between the two core cities Chengdu and Chongqing, which mainly benefits from the Chengdu Chongqing ring road The connection of expressways such as Chongqing railway, Chongqing Chengdu Expressway and Chengnan expressway has improved the traffic accessibility of Chengdu and Chongqing cities and the cities along the lines, and made them more closely connected; the formation of Zigong Neijiang dual core is mainly due to their geographical proximity and close communication ${ }^{[4]}$. This is also in line with the spatial structure of the "two circles and two belts" mentioned above. The traffic density at the nodes of the traffic network is far greater than that in the area with sparse network. The internal population conforms to the traffic network structure and forms a close relationship within the urban agglomeration. Whether the flow law of the elements in the region driven by the traffic flow pattern is consistent with the characteristics of the virus epidemic situation needs further analysis.

\subsection{Characteristics of Population Migration Flow in Urban Agglomeration}

"Report on the development of floating population in China 2018" points out that the outflow population within the Chengdu Chongqing urban agglomeration mainly flows into Chengdu and Chongqing, and the trend is more and more obvious. In order to study the relationship between virus transmission and population migration in Chengdu Chongqing urban agglomeration, the migration and flow of population in Chengdu Chongqing urban agglomeration during the latent period of epidemic outbreak were summarized as follows:

From January 10 to 24 is the peak period of spring transportation. Meishan city is the main destination of Chengdu City, accounting for 7.79\% of the total population; Deyang City, 7.71\% of the total population; Nanchong City, $6.87 \%$ of the total population. The top 10 destinations are Ziyang City and Chongqing city. Except Chongqing City, the rest are all cities in Sichuan Province. In the same period of time, the main destinations of Chongqing's emigration population are: Guang'an City, accounting for $10.36 \%$ of the total population of Chongqing; Chengdu City, accounting for $8.22 \%$ of the total population of Chongqing; Dazhou City, accounting for $6.79 \%$ of the total population of Chongqing. It can be seen that in recent years, the proportion of Sichuan and Chongqing flowing to the coastal developed areas is decreasing, while the population flow between the two core cities is increasing. In the two days from January 22 to 23, Chongqing accounted for $1.02 \%$ and Chengdu accounted for $0.25 \%$. The proportion of population flowing to the core city Chongqing was higher, and the distribution of epidemic situation was very similar to it.

\subsection{Characteristics of Infectious Virus Transmission in Urban Agglomerations}

According to the Chengdu Chongqing urban agglomerations, Chongqing and Chengdu are the two core cities, and conform to their traffic network. In the urban agglomeration, the curve 
continued to rise in the early stage, and reached the peak value in the middle period. After that, the epidemic situation was basically stable and the number of new cases was close to 0 . In the early stage, the epidemic situation in node cities such as Mianyang, Neijiang, Luzhou and other cities was significantly higher than that in other cities, and the color of the areas with large traffic flow was darker. In the later stage, due to the adoption of traffic regional control measures, the epidemic situation was basically controlled, showing a single digit growth, and the regional distribution changed little. At the same time, the emigration population of Chengdu was concentrated in Meishan and Germany The results show that there is a strong correlation between the network structure of urban agglomeration reflected by population traffic flow and migration flow and the regional epidemic situation of new coronavirus Relationship. In the geographical spatial distribution, it shows the clustering characteristics of "strong center sub center edge area", and has certain spillover characteristics. The division of risk prevention and control in urban agglomeration is basically consistent with the traffic network distribution of "two circles and two belts" and the trend of population migration.

\section{Analysis on Regional Joint Defense Mode of Urban Agglomerations}

In order to cut off the transmission path of the virus fundamentally, we need to further control the macro regional overall control, hierarchical prevention and control and joint prevention and control of relevant subjects' public participation in the regional space.

\subsection{Macro Regional and Hierarchical Prevention and Control}

Under the background of the outbreak of major health events, it is necessary to carry out joint prevention and control within urban agglomeration based on the internal structure of urban agglomeration. In response to this regional prevention and control, we should pay attention to the overall grasp from the macro level, and carry out hierarchical prevention and control according to the risk level. At the macro level, we should grasp the overall direction of epidemic prevention and control, closely monitor the population and traffic flow of the whole region, take preventive measures against floating population, implement control measures, isolate, observe and treat infectious disease patients and suspected patients on the spot, and take centralized or home-based medical observation on close contacts according to the situation to grasp the epidemic situation If necessary, it is necessary to take a level-1 public health response in time, and let the people's governments at all levels quickly organize and coordinate relevant units to participate in the handling of public health emergencies.

\subsection{Relevant Subjects Participate in Network Joint Defense}

If the urban agglomeration is regarded as a closely connected network, the silk line is the transportation network connecting the inner city group. The transportation oriented supporting system for convenient living, living and working can avoid the risk of over concentration of population and the occurrence of a series of "big city" diseases.

First of all, the government needs to strengthen cooperation and joint prevention. During the outbreak of the epidemic, the government quickly determined the overall layout of joint prevention and control, so that the prevention and control of the whole epidemic situation had a detailed deployment at the overall spatial level, and constantly adjusted according to the development of the situation. At the end of February, the large-scale spread situation within the Chengdu Chongqing urban agglomeration has been basically controlled. This is due to the emergency response of the central city and sub central city within the urban agglomeration and the timely cooperation of the surrounding cities.

Secondly, it is necessary to strengthen the prevention and control measures of residents and closely organize the epidemic prevention and control network. One is to widely publicize the emergency notice. The awareness rate of residents can be improved through television, Internet and road radio publicity. Second, set up special personnel to stick to the shed, set up traffic control points in densely populated road sections, adhere to the principle of strict control, and all personnel 
and vehicles can pass through with certificates. It can not only control the entry and exit of personnel, but also timely understand the situation of personnel and screen out useful information.

\subsection{Prevention and Control of Modern Technology}

In this prevention and control work, we should maximize the role of the Internet, and spread information through the Internet, so that residents can not only feel the severity of the epidemic situation, but also actively cooperate with the prevention and control of the epidemic situation. For example, the Internet should be used to continuously disclose the new cases, the trend of the epidemic situation, and the distribution of the epidemic situation. In the areas where the network is not smooth, it can be played on the TV station at a fixed time, At the same time, it can make use of the popularity of mobile phones to track the health code of the residents in the area, and only need to scan the code to check again to track the track of the residents in the area. In this way, the health code can be used to understand the flow trajectory of personnel at the first time, and the high-risk population can be screened out in time, and the low-risk people can be tracked and understood in real-time. The mobile phone health code can also be used as the first pass for screening in and out of major public places, so as to eliminate the risk of personnel entering the personnel gathering places in time.

\section{Conclusion}

Henri Lefebvre, a western scholar, thinks that urban agglomeration is the carrier of the relationship between cities. They are closely linked and have a certain humanistic foundation ${ }^{[6]}$. Therefore, in the face of any event, the flow of elements within urban agglomerations can be regarded as one aspect of control. The Chengdu Chongqing urban agglomeration discussed in this paper is an important urban community derived from Southwest China in recent years, which has a good performance in the prevention and control of this major health event. Although the huge traffic flow and population migration flow caused by the large number of population moving towards the core cities and important cities in the early stage brought great difficulties to the joint prevention and control, the epidemic situation was still controlled in the minimum range.

In fact, the joint control mechanism between cities will become more and more efficient in the future. In view of the limited data, this paper only analyzes the characteristics of the virus transmission through the characteristics of traffic flow and population flow in urban agglomeration, and puts forward epidemic prevention measures and joint prevention and control measures in the later stage. It is expected that the operation of joint prevention and control mechanism in urban agglomeration and the causes of epidemic outbreak in Ganzi area can be deeply studied in the future.

\section{References}

[1] Zheng Kejia, Ma Rongjun. Manuel Castel and flow space theory. Urban and rural planning, landscape architecture and greening, Vol.27, No.12, pp.60-62, 2009.

[2] Cen Di, Zhou Jianyun, Zhao Miaoxi. Research on new urbanization from the perspective of “flow space”. Planners forum, Vol.29, No.4, pp.15-20, 2013.

[3] Guo Rong, Ruan Yuyao. Study on urban network characteristics of Heilongjiang Province Based on traffic flow space. Architectural design and urban rural planning, Vol.2, No.8, pp.24-29, 2020

[4] Li Zhengrong, Xu Deng, Yao Wenyu Zhao. Analysis of urban network structure characteristics and organization mode from the perspective of flow space -- Based on the comparison of traffic flow and information flow of Chengdu Chongqing urban agglomeration. Economic Forum, Vol.2, No.8, pp.143-149, 2018. 